TRANSACTIONS OF THE

AMERICAN MATHEMATICAL SOCIETY

Volume 352, Number 8, Pages 3473-3493

S 0002-9947(00)02562-9

Article electronically published on April 17, 2000

\title{
MORSE THEORY FOR THE YANG-MILLS FUNCTIONAL VIA EQUIVARIANT HOMOTOPY THEORY
}

\author{
URSULA GRITSCH
}

\begin{abstract}
In this paper we show the existence of non-minimal critical points of the Yang-Mills functional over a certain family of 4-manifolds $\left\{M_{2 g}: g=\right.$ $0,1,2, \ldots\}$ with generic $S U(2)$-invariant metrics using Morse and homotopy theoretic methods. These manifolds are acted on fixed point freely by the Lie group $S U(2)$ with quotient a compact Riemann surface of even genus. We use a version of invariant Morse theory for the Yang-Mills functional used by Parker in A Morse theory for equivariant Yang-Mills, Duke Math. J. 66-2 (1992), 337-356 and Råde in Compactness theorems for invariant connections, submitted for publication.
\end{abstract}

\section{INTRODUCTION}

In this paper we construct a family of Riemannian spin 4-manifolds, denoted by $\left\{M_{2 g}: g=0,1,2, \ldots\right\}$, acted on by the Lie group $S U(2)$ and prove an existence result for non-minimal critical points of the Yang-Mills functional over the manifold $M_{2 g}$ (for $g \neq 1$ ) for generic $S U(2)$-invariant metrics. The manifold $M_{2 g}$ is the product of a compact Riemann surface $\mathcal{F}_{2 g}$ of even genus and the two-sphere $S^{2}$ modulo an involution. The $S U(2)$-action is the standard action on $S^{2}$ and the trivial action on $\mathcal{F}_{2 g}$. In section 3.4 we prove

Theorem 3.4.10. Fix $g=0,1,2, \ldots$ and let $\Delta^{+}$and $\Delta^{-}$be the positive and negative $S U(2)$-equivariant spinor bundles over the 4-manifold $M_{2 g}$. Fix an $S U(2)$ invariant metric.

(i) The Yang-Mills functional restricted to the invariant orbit space $\mathcal{Y} M: \mathcal{B}_{S U(2)}$ $\rightarrow \mathbb{R}$ over the manifold $M_{2 g}$ has at least $2 g+1$ critical points on each of the bundles $\Delta^{+}$and $\Delta^{-}$.

(ii) In the case $g=0$ for a generic $S U(2)$-invariant metric the critical point on the bundle $\Delta^{+}$cannot be self dual and on the bundle $\Delta^{-}$it cannot be anti-self dual. In the case $g \geq 2$ for a generic $S U(2)$-invariant metric at least one of the critical points on the bundle $\Delta^{+}$cannot be anti-self dual and at least one of the critical points on the bundle $\Delta^{-}$cannot be self dual.

Received by the editors March 24, 1998 and, in revised form, September 20, 1998.

2000 Mathematics Subject Classification. Primary 58E15, 55P91.

Key words and phrases. Non-minimal critical points, Yang-Mills, equivariant gauge theory, equivariant homotopy theory.

This note is part of my Ph.D. thesis written at Stanford University, 1997. I thank my advisor Ralph Cohen for constant support and encouragement and the Studienstifung des deutschen Volkes for a dissertation fellowship. Part of this paper was written while the author was supported by an EPSRC-fellowship at DPMMS, Cambridge, U.K. 
By Palais' principle of symmetric criticality [Pal2] each of the critical points in (i) is a critical point of the Yang-Mills functional on the non-equivariant orbit space $\mathcal{Y} M: \mathcal{B} \rightarrow \mathbb{R}$, i.e. they are Yang-Mills connections.

For a long time it was conjectured that by analogy with the harmonic map problem on the two-sphere the Yang-Mills functional on the 4-sphere does not have other critical points besides the self dual and anti-self dual connections. In SSU] L.M. Sibner, R.J. Sibner and K.K. Uhlenbeck, however, construct a sequence of non-minimal critical points on the trivial $S U(2)$-bundle on the 4-sphere with the round metric. In [SS L. Sadun and J. Segert find a critical point of the Yang-Mills functional on every $S U(2)$-principal bundle $\eta$ over the 4 -sphere $S^{4}$ with the standard metric provided its second Chern class $c_{2}(\eta)$ is not equal to \pm 1 . In Wan H.-Y. Wang proves the existence of an infinite number of irreducible $S U(2)$-connections over the manifolds $S^{2} \times S^{2}$ and $S^{1} \times S^{3}$ with the standard metrics which are nonminimal solutions to the Yang-Mills equations. Finally in [Pa2] T.H. Parker proves the existence of irreducible non-minimal Yang-Mills fields on the trivial bundle over $S_{L}^{1} \times S^{3}$ for some $L$, where $S_{L}^{1}$ denotes the circle of radius $L$; and he shows that there exists a family of metrics on the 4-sphere $S^{4}$ each of which admits an irreducible Yang-Mills field on the trivial $S U(2)$-bundle.

Naturally one would like to do Morse theory for the Yang-Mills functional defined on the space of connections on a principle bundle over a Riemannian 4-manifold modulo the action of the gauge group. Since this space $\mathcal{B}$ is infinite dimensional, the Yang-Mills functional needs to satisfy a certain compactness condition, called the Palais-Smale condition. However, by Uhlenbeck's weak compactness theorem, it is well-known that the Yang-Mills functional defined on the orbit space $\mathcal{B}$ does not satisfy this condition.

As noted in [Pa1] this problem does not occur if the 4-manifold $M$ is acted on by a compact Lie group $H$ such that the action has no zero dimensional orbits and we restrict the Yang-Mills functional to the orbit space $\mathcal{B}_{H}$ of $H$-invariant connections modulo the $H$-invariant gauge group.

A proof was outlined in [Pa1] that the Yang-Mills functional defined on the invariant orbit space $\mathcal{B}_{H}$ satisfies the Palais-Smale condition provided the orbit space $\mathcal{B}_{H}$ has no singularities. Unfortunately this proof contains a gap: It is not clear that one can choose good invariant gauges. A different proof was given recently by J. Råde in [Rã]. (See also section 3.1 of this paper.)

In [Pa1] Parker uses his Morse theory to reprove Sadun's and Segert's result in $\left[\mathrm{SS}\right.$ using a fixed point free $S U(2)$-action on $S^{4}$ called the quadrupole action. There is also a fixed point free $S U(2)$-action on $S^{2} \times S^{2}$ but Parker's Morse theory is too weak to establish the existence of irreducible non-(anti)-self dual Yang-Mills connections. The manifold $M_{2 g}$ in theorem 3.4.10 is acted on fixed point freely by the Lie group $S U(2)$, too.

The general strategy to prove theorem 3.4 .10 as proposed by Parker in [Pa1] is as follows. Let $\mathcal{M}_{H} \hookrightarrow \mathcal{B}_{H}$ denote the subspace of the absolute minima of the Yang-Mills functional modulo the invariant gauge group. We assume that the second Chern class of the bundle we are working with is positive. Then the absolute minima of the Yang-Mills functional are the anti-self dual connections and there are no self dual connections. (By changing the orientation we obtain the opposite case of negative second Chern class). If the inclusion $\iota: \mathcal{M}_{H} \hookrightarrow \mathcal{B}_{H}$ is not a homotopy equivalence, then the Palais-Smale condition for the Yang-Mills functional implies the existence of at least one non-minimal critical point of the Yang-Mills functional 
on the space $\mathcal{B}_{H}$. For otherwise the retraction along the flow lines of the gradient flow of the Yang-Mills functional would give a homotopy inverse of the inclusion $\iota: \mathcal{M}_{H} \hookrightarrow \mathcal{B}_{H}$.

It follows from Uhlenbeck's weak convergence theorem and its equivariant versions in $\mathrm{Cho}$ and $\mathrm{Ba}$ ] that if the bundle $\eta$ does not admit a reducible $H$-invariant anti-self dual connection for any metric, then for a generic $H$-invariant metric the moduli space $\mathcal{M}_{H}$ is a (possibly empty) compact closed manifold of a dimension which can be computed using the equivariant Atiyah-Singer index theorem.

Denote by $\mathcal{B}_{H}^{0}$ the based invariant orbit space of invariant connections modulo the based invariant gauge group. In section 2 of this paper we identify the weak homotopy type of the space $\mathcal{B}_{H}^{0}$ in terms of an invariant mapping space. This is a direct generalization from the corresponding non-equivariant theorem in $\mathrm{AB}$, prop. 2.4, p. 540] or [DK, prop. 5.1.4, p. 174]. It will turn out that in our example of theorem 3.4.10 (for $M=M_{2 g}$ and $H=S U(2)$ ) the orbit spaces $\mathcal{B}_{H}$ and $\mathcal{B}_{H}^{0}$ are homeomorphic. Then the information on the homotopy type of the space $\mathcal{B}_{H}$ and the dimension of the manifold $\mathcal{M}_{H}$ for generic $H$-invariant metrics will be enough to deduce that the inclusion $\mathcal{M}_{H} \hookrightarrow \mathcal{B}_{H}$ cannot be a homotopy equivalence.

In section 3.2 we define the manifolds $M_{2 g}$ for $g=0,1,2, \ldots$ acted on fixed point freely by the Lie group $S U(2)$, show that they are spin and compute the second Chern classes of the positive and negative spinor bundles $\Delta^{+}$and $\Delta^{-}$. In section 3.3 we compute the homotopy type of the invariant orbit space $\mathcal{B}_{S U(2)}$ and in section 3.4 we prove theorem 3.4.10.

\section{The HOMOtopy type of THE SPACE $\mathcal{B}_{H}^{0}$}

2.1 Notation and terminology. Let $M$ be a closed oriented Riemannian 4manifold and let $\eta=(P \rightarrow M)$ be an $S U(2)$-principal bundle over the manifold $M$. Denote by $\mathcal{A}$ the space of smooth connections on the bundle $\eta$. Let $\mathcal{G}$ be the group of smooth gauge transformations of this bundle. This means that elements $g \in \mathcal{G}$ are smooth automorphisms of the bundle $\eta$. Let $\mathcal{G}^{0}$ be the subgroup of the group $\mathcal{G}$ of gauge transformations whose elements are the identity over a given base point $m \in M$.

Let $H$ be a compact Lie group acting smoothly on the manifold $M$ on the left. We also assume that the action lifts to the total space $P$ of the bundle $\eta$ such that the left action of the Lie group $H$ on the total space $P$ commutes with the right action of the structure group $S U(2)$ on $P$. We define the invariant gauge group $\mathcal{G}_{H}$ to be the subgroup of the gauge group $\mathcal{G}$ such that each gauge transformation commutes with the action of the Lie group $H$ on the total space $P$ of the bundle $\eta$. Analogously we define the based invariant gauge group $\mathcal{G}_{H}^{0}$ to be the equivariant gauge transformations which are the identity over a given based orbit $\mathcal{O}_{m} \subset M$.

The groups $\mathcal{G}$ and $\mathcal{G}^{0}$ act naturally on the space of connections $\mathcal{A}$ from the right by the pull back of connections. We define the orbit space $\mathcal{B}$ to be the space $\mathcal{A} / \mathcal{G}$ of connections modulo the action of the gauge group and the based orbit space $\mathcal{B}^{0}$ to be the space $\mathcal{A} / \mathcal{G}^{0}$ of connections modulo the action of the based gauge group.

The left action of the group $H$ on the bundle $\eta$ induces a right action on the space of connections $\mathcal{A}$ again by the pull back of connections. The fixed points of this action are called $H$-invariant connections. We denote the space of fixed points by $\mathcal{A}_{H}$. The invariant groups $\mathcal{G}_{H}$ and $\mathcal{G}_{H}^{0}$ act on the space of invariant connections $\mathcal{A}_{H}$ as above. As in the non-equivariant setting we define the invariant orbit space 
$\mathcal{B}_{H}$ to be the space $\mathcal{A}_{H} / \mathcal{G}_{H}$ of invariant connections modulo the invariant gauge group and the based invariant orbit space $\mathcal{B}_{H}^{0}$ to be the space $\mathcal{A}_{H} / \mathcal{G}_{H}^{0}$ of invariant connections modulo the based invariant gauge group.

It is customary in gauge theory to complete the space $\mathcal{A}$ in the $L^{2,2}$-Sobolev norm and the gauge group $\mathcal{G}$ in the $L^{3,2}$-Sobolev norm using a fixed connection $A$ on the bundle $\eta$. For notational convenience we still denote the completed space of connections by $\mathcal{A}$ and the completed gauge group by $\mathcal{G}$. Then the group $\mathcal{G}$ is a Hilbert Lie group which acts smoothly on the space of connections $\mathcal{A}$. Analogously we complete the space of invariant connections $\mathcal{A}_{H}$ in the $L^{2,2}$-Sobolev norm and the invariant gauge group $\mathcal{G}_{H}$ in the $L^{3,2}$-norm using an $H$-invariant connection which we fix once and for all. The based gauge group $\mathcal{G}^{0}$ is a closed subgroup (in the $L^{3,2}$-norm) of the full gauge group $\mathcal{G}$. The same holds for the based invariant gauge group $\mathcal{G}_{H}^{0}$. The orbit spaces $\mathcal{B}$ and $\mathcal{B}^{0}$ and the invariant orbit spaces $\mathcal{B}_{H}$ and $\mathcal{B}_{H}^{0}$ inherit a topology from the $L^{2,2}$-norm on the space of connections $\mathcal{A}$ or the space of invariant connections $\mathcal{A}_{H}$.

The results of section 2 are also true if we complete the spaces $\mathcal{A}$ and $\mathcal{A}_{H}$ using Morrey norms as used in [Ra]. This will be used in section 3 and explained in 3.1. The reader can also take the spaces $\mathcal{B}$ and $\mathcal{B}_{H}$ to be the smooth (invariant) connections modulo the smooth (invariant) gauge group together with a topology induced from Sobolev or Morrey norms on the space of smooth (invariant) connections.

2.2 Classification of equivariant bundles. In order to get a model for the space $\mathcal{B}_{H}^{0}$ in terms of an equivariant mapping space we need to classify equivariant principal bundles. These are classified in much the same way as ordinary bundles are. For computational reasons which will become clear later we prefer to describe the classification theory of equivariant vector bundles. However the classification theories of equivariant vector bundles and equivariant principal bundles are really equivalent. We only give definitions and state results.

Let $V$ be a fixed unitary $H$-representation. Let $G_{n}\left(V^{k}\right)$ be the Grassmannian of unitary $n$-planes in the vector space $V^{k}=\underbrace{V \oplus \cdots \oplus V}_{k \text { times }}$ for $k>n$. The group $H$ acts naturally on the space $G_{n}\left(V^{k}\right)$ because it acts on the space $V^{k}$. We denote by $G_{n}\left(V^{\infty}\right)=\lim _{k \rightarrow \infty} G_{n}\left(V^{k}\right)$ the direct limit as an $H$-equivariant space. Let $\gamma_{n}\left(V^{k}\right)=\left(E_{n}\left(V^{k}\right) \rightarrow G_{n}\left(V^{k}\right)\right)$ denote the canonical unitary bundle over the Grassmannian. The fiber over a plane $P \in G_{n}\left(V^{k}\right)$ are the points $p \in P$. The $H$-action on the Grassmannian lifts naturally to an $H$-action on the total space of the canonical bundle giving this bundle the structure of an $H$-equivariant bundle. We take the limit $E_{n}\left(V^{\infty}\right)=\lim _{k \rightarrow \infty} E_{n}\left(V^{k}\right)$ and obtain the $H$-equivariant bundle $\gamma_{n}\left(V^{\infty}\right)=\left(\pi: E_{n}\left(V^{\infty}\right) \rightarrow G_{n}\left(V^{\infty}\right)\right)$.

Define $\operatorname{Vect}_{H}^{V, n}(M)$ to be the isomorphism classes of $H$-equivariant unitary $n$ dimensional vector bundles over $M$ with the following property: For every $m \in M$ the isotropy representation of the isotropy group $H_{m}$ on the fiber over $m$ is equivalent to a sub-module of the $H_{m}$-module $V^{n}$ induced by the given $H$-module $V$. We call such vector bundles "subordinate to the representation $V$ ". Let $\left[M, G_{n}\left(V^{\infty}\right)\right]^{H}$ denote the $H$-equivariant homotopy classes of the $H$-maps from the manifold $M$ to the equivariant Grassmannian $G_{n}\left(V^{\infty}\right)$.

Theorem 2.2.1 (Wasserman, Segal). The map

$$
\begin{aligned}
{\left[M, G_{n}\left(V^{\infty}\right)\right]^{H} } & \rightarrow \operatorname{Vect}_{H}^{V, n}(M) \\
{[f] } & \mapsto\left(f^{*}\left(\gamma_{n}\left(V^{\infty}\right)\right)\right)
\end{aligned}
$$


is well defined, natural in $M$ and an isomorphism. The same classification theorem holds in the case of orthogonal or symplectic equivariant vector bundles if we choose the $H$-module $V$ to be orthogonal or symplectic.

Proof. A proof can be found in Wa, section 2, p. 132] in the case of orthogonal equivariant vector bundles. The same proof carries over to unitary and symplectic equivariant vector bundles. A proof of a similar result in the case of complex vector bundles over a compact manifold is also given in [Se, section 1, p. 131].

We need a similar classification theorem for bundles with a fixed equivariant trivialization over a fixed orbit. Fix one orbit $\mathcal{O}_{m} \cong H / H_{m}$ for some $m \in M$ once and for all. Let $\operatorname{Vect}_{H, 0}^{V, n}(M)$ denote the set of isomorphism classes of equivariant $n$-dimensional unitary vector bundles over $M$ subordinate to the representation $V$ together with a fixed trivialization over the orbit $\mathcal{O}_{m}$. Fix a point $* \in G_{n}\left(V^{\infty}\right)$. Let $\left[M, G_{n}\left(V^{\infty}\right)\right]_{0}^{H}$ denote the equivariant pointed homotopy classes of $H$-maps $f: M \rightarrow G_{n}\left(V^{\infty}\right)$ which map the point $m$ to the chosen point $* \in G_{n}\left(V^{\infty}\right)$ and hence which map the orbit $\mathcal{O}_{m} \subset M$ to the orbit $\mathcal{O}_{*} \subset G_{n}\left(V^{\infty}\right)$.

Theorem 2.2.2. Assume that the manifold $M$ is compact. There is a point $* \in$ $G_{n}\left(V^{\infty}\right)$ such that the map

$$
\begin{aligned}
{\left[M, G_{n}\left(V^{\infty}\right)\right]_{0}^{H} } & \rightarrow \operatorname{Vect}_{H, 0}^{V, n} \\
{[f] } & \mapsto\left(f^{*}\left(\gamma_{n}\left(V^{\infty}\right)\right)\right)
\end{aligned}
$$

is well defined, natural in $M$ and an isomorphism. The same classification theorem holds in the case of orthogonal or symplectic equivariant vector bundles if we choose the $H$-module $V$ to be orthogonal or symplectic.

Proof. The proof of theorem 2.2.2 uses the same methods as the proof of theorem 2.2.1 working in the category of based equivariant vector bundles.

2.3 The weak homotopy equivalence $\mathcal{B}_{H}^{0} \simeq \operatorname{Map}_{H}^{0}(M, B(H, S p(1)))^{\eta}$. Recall that $\eta=(P \rightarrow M)$ is an $H$-equivariant $S U(2) \cong S p(1)$-principal bundle and we also denote by $\eta=\left(E=P \times_{S p(1)} \mathbb{H} \rightarrow M\right)$ the associated quaternionic line bundle with structure group $S U(2) \cong S p(1)$. Choose an orbit $\mathcal{O}_{m} \cong H / H_{m}$ in $M$ and a fixed trivialization of the bundle $\eta$ over $\mathcal{O}_{m}$. Choose a quaternionic representation $V$ of the group $S p(1)$ such that the vector bundle $\eta$ is subordinate to $V$. Denote by $B(H, S p(1))=G_{1}\left(V^{\infty}\right)$ the corresponding Grassmannian which classifies the vector bundle $\eta$ together with the trivialization over the orbit $\mathcal{O}_{m}$ according to theorem 2.2.2. In the following, if an $H$-equivariant vector bundle $\xi$ is subordinate to an $H$-module $V$, we say that the associated principle frame bundle (also denoted by $\xi$ ) is subordinate to the $H$-module $V$ as well. Also, throughout this paper, we give all spaces of maps the compact-open topology.

To prove the weak homotopy equivalence

$$
\mathcal{B}_{H}^{0} \simeq \operatorname{Map}_{H}^{0}(M, B(H, S p(1)))^{\eta}
$$

(where the right hand side denotes the component of the mapping space of maps which classify the bundle $\eta$ ) we follow the proof of the corresponding non-equivariant result

$$
\mathcal{B}^{0} \simeq \operatorname{Map}^{0}(M, B(S p(1)))^{\eta}
$$

in DK, prop. 514, p. 174]. 
As in $[\mathrm{DK}$ (5.1.5), p. 175] associated to the $H$-equivariant $S U(2)$-principal bundle $\eta=(P \rightarrow M)$ we have the bundle $\Xi$ over the base $\mathcal{B}_{H}^{0} \times M$ defined as $S U(2) \rightarrow \mathcal{A}_{H} \times_{\mathcal{G}_{H}^{0}} P \rightarrow \mathcal{B}_{H}^{0} \times M$. The bundle $\Xi$ is in a natural way an $H$-equivariant bundle and hence it is classified by an $H$-equivariant map $\delta: \mathcal{B}_{H}^{0} \times M \rightarrow G_{1}\left(V^{\infty}\right)=$ $B(H, S p(1)$ ). Here $V$ is an $H$-module to which the bundle $\eta$ (and therefore also the bundle $\Xi)$ is subordinate.

Theorem 2.3.1. The adjoint of the map $\delta$

$$
\delta^{a d}: \mathcal{B}_{H}^{0} \rightarrow \operatorname{Map}_{H}^{0}(M, B(H, S p(1)))^{\eta}
$$

is well-defined and induces a weak homotopy equivalence.

Proof. The proof is a technical modification of the analogous result in the nonequivariant setting given in [DK, prop. 5.1.4, p. 174]. One uses a universal family of $H$-invariant framed connections on the bundle $\Xi$.

\section{Non-minimal CRitical points of the Yang-Mills functional}

In the remaining part of this paper we construct the family $\left\{M_{2 g}: g=0,1,2, \ldots\right\}$ of 4-manifolds and prove theorem 3.4.10.

3.1 Analytical background. We introduce certain completions of the spaces $\mathcal{A}$, $\mathcal{A}_{H}, \mathcal{G}$ and $\mathcal{G}_{H}$ not usually used in gauge theory but used in [Ra]. Using these norms J. Råde is able to show ([Rå, Theorems 3 and 4, p. 4]) that the Yang-Mills functional on the space $\mathcal{B}_{H}=\mathcal{A}_{H} / \mathcal{G}_{H}$ satisfies the Palais-Smale condition provided the group $H$ acts isometrically with no zero dimensional orbits on the manifold $M$ and the $H$-equivariant principal bundle $\eta=(S U(2) \rightarrow P \rightarrow M)$ has no reducible connections. (A connection is called reducible if its isotropy group of the action of the gauge group on the space of connections is larger than the subgroup $\mathbb{Z}_{2}=\{ \pm 1\}$ of constant gauge transformations).

The Morrey space $L_{\lambda}^{p}\left(\mathbb{R}^{n}\right)=L_{\lambda}^{0, p}\left(\mathbb{R}^{n}\right)$, with $p \in[1, \infty)$ and $\lambda \in \mathbb{R}$, is defined as the space of all $f \in L^{p}\left(\mathbb{R}^{n}\right)$ such that

$$
\sup _{\rho \in(0,1]} \sup _{x \in \mathbb{R}^{n}} \rho^{\lambda-n}\|f\|_{L^{p}\left(B_{\rho}(x)\right)}^{p}<\infty .
$$

It is a Banach space with norm

$$
\|f\|_{L_{\lambda}^{p}\left(\mathbb{R}^{n}\right)}^{p}=\|f\|_{L^{p}\left(\mathbb{R}^{n}\right)}^{p}+\sup _{\rho \in(0,1]} \sup _{x \in \mathbb{R}^{n}} \rho^{\lambda-m}\|f\|_{L^{p}\left(B_{\rho}(x)\right)}^{p} .
$$

The Morrey space $L_{\lambda}^{k, p}\left(\mathbb{R}^{n}\right)$ with $k$ a positive integer, $p \in[1, \infty)$ and $\lambda \in \mathbb{R}$, is defined as the space of all $f \in L_{\lambda}^{k, p}\left(\mathbb{R}^{n}\right)$ such that $\partial^{\alpha} f \in L_{\lambda}^{p}\left(\mathbb{R}^{n}\right)$ for all $\alpha$ with $|\alpha| \leq k$. It is a Banach space with norm

$$
\|f\|_{L_{\lambda}^{k, p}\left(\mathbb{R}^{n}\right)}^{p}=\sum_{|\alpha| \leq k}\left\|\partial^{\alpha} f\right\|_{L_{\lambda}^{p}\left(\mathbb{R}^{n}\right)}^{p} .
$$

The global Morrey spaces $L_{\lambda}^{k, p}(M)$ are defined using a local trivialization on the manifold $M$ ([Rå, p. 10]).

The reason why Morrey spaces are useful in equivariant gauge theory are the following two observations made in [Rå. 
Lemma 3.1.1 (Special case of [Rå, Lemma A.1, p. 18]). Let $H$ be a compact Lie group that acts smoothly on the manifold $M$, in such a way that all $H$-orbits have dimension $\geq 1$, and that acts smoothly on the bundle $\eta$. If $s \in L^{k, p}(M, \eta)$ with $k \in \mathbb{Z}$ and $p \in[1, \infty)$, is $H$-invariant, then $s \in L_{s}^{k, p}(M, \eta)$. If $A$ is an $H$-invariant connection on $\eta$, then

$$
\|s\|_{L_{3, A}^{k, p}(M)}^{p} \leq c\|s\|_{L_{A}^{k, p}(M)}^{p} .
$$

The constant $c$ only depends on the Riemannian manifold $M$ and on the orbits of the action of the group $H$ on the manifold $M$.

The second point is that the Morrey spaces $L_{d}^{k, p}$ in $n$ dimensions satisfy "the same" embedding theorems as the Sobolev spaces $L^{k, p}$ in $d$ dimensions. Hence we get multiplications

$$
\begin{aligned}
& L_{3}^{2,2} \times L_{3}^{2,2} \rightarrow L_{3}^{2,2} \\
& L_{3}^{2,2} \times L_{3}^{1,2} \rightarrow L_{3}^{1,2} \\
& \text { and } \quad L_{3}^{1,2} \times L_{3}^{1,2} \rightarrow L_{3}^{3} \rightarrow L^{2} \quad(\text { see }[\text { Rå, p. 12] }) .
\end{aligned}
$$

It follows that if we complete the gauge groups $\mathcal{G}$ and $\mathcal{G}_{H}$ and the spaces $\mathcal{A}$ and $\mathcal{A}_{H}$ in the $L_{3}^{2,2}$ - and $L_{3}^{1,2}$-norm respectively, then $\mathcal{G}$ and $\mathcal{G}_{H}$ are Hilbert Lie groups that act smoothly on $\mathcal{A}$ or $\mathcal{A}_{H}$ and the Yang-Mills functional is continuous on $\mathcal{A}$ and $\mathcal{A}_{H}$.

J. Råde is now able to show in [Rå Theorems 3 and 4, p. 4] that the Yang-Mills functional on the space $\mathcal{B}_{H}=\mathcal{A}_{H} / \mathcal{G}_{H}$ satisfies the Palais-Smale condition provided the group $H$ acts isometrically with no zero dimensional orbits on the manifold $M$ and the $H$-equivariant principal bundle $\eta=(S U(2) \rightarrow P \rightarrow M)$ has no reducible connections.

If the bundle $\eta$ does not admit reducible connections the invariant orbit space $\mathcal{B}_{H}$ is an infinite dimensional Hilbert manifold. The $L^{1,2}$-metric on the space of invariant connections $\mathcal{A}_{H}$ is $\mathcal{G}_{H}$-invariant and descends to a metric on the manifold $\mathcal{B}_{H}$. Lemma 3.1.1 shows that the space $\mathcal{B}_{H}$ together with the $L_{3}^{1,2}$-topology and the $L^{1,2}$-metric is a complete Riemannian manifold.

3.2 The manifold $M_{2 g}=\mathcal{F}_{2 g} \times_{\sigma} S(v)$. Let $\mathcal{F}_{2 g}$ denote an oriented Riemann surface of genus $2 g$. We denote by $S(v)$ the unit sphere in the representation $v$ where $v$ is the standard representation of the Lie group $S O(3)$ on $\mathbb{R}^{3}$. The space $S(v)$ has an induced $S O(3)$-action. Topologically the represenation sphere $S(v)$ is just the 2-sphere $S^{2}$. We define an involution $\sigma$ on the product $\mathcal{F}_{2 g} \times S(v)$ as follows. We think of the Riemann surface $\mathcal{F}_{2 g}$ as being obtained from two oriented Riemann surfaces $\mathcal{F}_{g}$ of genus $g$ with a 2 -disk $D^{2}$ removed and glued along the boundary $S^{1}$, i.e.

$$
\mathcal{F}_{2 g}=\left(\mathcal{F}_{g}-D^{2}\right) \cup_{S^{1}}\left(\mathcal{F}_{g}-D^{2}\right) .
$$

We say that one of the surfaces $\mathcal{F}_{g}-D^{2}$ is positive and think of it as lying above the circle $S^{1}$. We call the other negative, think of it as lying below the circle $S^{1}$ and as the mirror image of the positive one. Then there is an involution

$$
\sigma_{1}: \mathcal{F}_{2 g} \rightarrow \mathcal{F}_{2 g}
$$

fixing the "gluing circle" $S^{1}$ and interchanging the positive and negative surfaces. 
Example 3.2.2. In the case $g=0$ the involution $\sigma_{1}$ is the map

$$
\begin{aligned}
\sigma_{1}: S^{2} & \rightarrow S^{2}, \\
\sigma_{1}(x, y, z) & =(x, y,-z) .
\end{aligned}
$$

Let $\sigma_{2}: S(v) \rightarrow S(v)$ be the reflection through the origin, i.e., $\sigma_{2}(x, y, z)=$ $(-x,-y,-z)$. Then we define

$$
\begin{aligned}
\sigma: \mathcal{F}_{2 g} \times S(v) & \rightarrow \mathcal{F}_{2 g} \times S(v) \\
\text { by } \quad \sigma(v, w) & =\left(\sigma_{1}(v), \sigma_{2}(w)\right) .
\end{aligned}
$$

Since the involutions $\sigma_{1}$ and $\sigma_{2}$ are both orientation reversing, the involution $\sigma$ : $\mathcal{F}_{2 g} \times S(v) \rightarrow \mathcal{F}_{2 g} \times S(v)$ is orientation preserving. Also since the involution $\sigma: \mathcal{F}_{2 g} \times S(v) \rightarrow \mathcal{F}_{2 g} \times S(v)$ has no fixed points, the quotient

$$
M_{2 g}=\mathcal{F}_{2 g} \times{ }_{\sigma} S(v)
$$

is a closed oriented 4-manifold. Any pair of metrics on the two-sphere $S(v)$ invariant under the involution $\sigma_{2}$ and on the Riemann surface $\mathcal{F}_{2 g}$ invariant under the involution $\sigma_{1}$ induce a metric on the manifold $M_{2 g}$. We fix one arbitrary metric on the manifold $M_{2 g}$.

The action of the Lie group $S O(3)$ on the sphere $S(v)$ commutes with the reflection through the origin in $S(v)$ and hence we obtain an $S O(3)$-action on the manifold $M_{2 g}$ by letting the group $S O(3)$ act trivially on the surface $\mathcal{F}_{2 g}$ and by the action on $S(v)$. For technical reasons which will become clear later we consider the induced $S U(2)$-action on the manifold $M_{2 g}$. This action has no fixed points as $S O(3)$ acts transitively on $S(v)$. Also since the group $S U(2)$ is connected it acts in an orientation preserving fashion on the manifold $M_{2 g}$. Since the group $S U(2)$ is compact we can assume that the chosen metric on the manifold $M_{2 g}$ is $S U(2)$-invariant.

Lemma 3.2.5. The manifold $M_{2 g}=\mathcal{F}_{2 g} \times_{\sigma} S(v)$ is a spin manifold.

Proof. Let $T M_{2 g}$ denote the tangent bundle of the manifold $M_{2 g}$. We have to show that the second Stiefel-Whitney class

$$
\omega_{2}\left(T M_{2 g}\right) \in H^{2}\left(M_{2 g} ; \mathbb{Z}_{2}\right)=\operatorname{Hom}\left(H_{2}\left(M_{2 g} ; \mathbb{Z}_{2}\right) ; \mathbb{Z}_{2}\right)
$$

is zero. Let $\left\{x_{i}: i \in I\right\} \subset H_{2}\left(M_{2 g} ; \mathbb{Z}_{2}\right)$ be a generating set of $H_{2}\left(M_{2 g} ; \mathbb{Z}_{2}\right)$ and represent every element $x_{i}$ by a map $f_{i}: X \rightarrow M_{2 g}$ where $X$ is a compact surface. It is enough to show that for every map $f_{i}$ as above the pull back bundle $f_{i}^{*}\left(T M_{2 g}\right)$ is trivial. We now only sketch the argument.

The group $H_{2}\left(M_{2 g} ; \mathbb{Z}_{2}\right) \cong H_{2}\left(M_{2 g} ; \mathbb{Z}\right) \otimes \mathbb{Z}_{2} \oplus \operatorname{Tor}\left(H_{1}\left(M_{2 g} ; \mathbb{Z}\right) ; \mathbb{Z}_{2}\right) \cong \mathbb{Z}_{2} \oplus \mathbb{Z}_{2}$ is generated by two cycles $f_{1} e_{1}^{+}$and $f_{0} e_{2}^{+}$chosen as follows. On the two-sphere $S(v)$ we choose a CW-decomposition symmetric under the reflection through the origin consisting of two 0-cells $e_{0}^{+}$and $e_{0}^{-}$, two 1-cells $e_{1}^{+}$and $e_{1}^{-}$and two 2-cells $e_{2}^{+}$ and $e_{2}^{-}$. The cell $f_{0}$ denotes a 0 -cell on the Riemann surface $\mathcal{F}_{2 g}$ which lies on the "gluing circle". Then $f_{1} e_{1}^{+}$and $f_{0} e_{2}^{+}$denote the images of the cartesian products of the appropriate cells on the Riemann surface $\mathcal{F}_{2 g}$ and the two sphere $S(v)$ under the projection $\pi: \mathcal{F}_{2 g} \times S(v) \rightarrow \mathcal{F}_{2 g} \times{ }_{\sigma} S(v)=M_{2 g}$.

The map $f: \mathbb{R} P^{2} \rightarrow \mathcal{F}_{2 g} \times_{\sigma} S(v)=M_{2 g}$ given by $[x] \mapsto\left[f_{0}, x\right]$ represents the torsion element $f_{0} e_{2}^{+}$. One shows that $f^{*}\left(T M_{2 g}\right)$ is the trivial bundle using the same ideas as in [MS Lemma 3.4, p. 43] to compute the tangent bundle of the manifold $\mathbb{R} P^{2}$. 
Let $g: S^{1} \times \mathbb{R} P^{1} \rightarrow \mathcal{F}_{2 g} \times_{\sigma} S(v)$ be the map $g(\lambda,[\mu])=[\lambda, \mu]$ where we identify the circle $S^{1}$ with the "gluing circle" $S^{1} \subset \mathcal{F}_{2 g}$. The map $g$ is a representative for the element $f_{1} e_{1}^{+}$. One shows that $g^{*}\left(T M_{2 g}\right) \cong S^{1} \times f^{*}\left(T M_{2 g}\right) / \mathbb{R} P^{1}$ where we consider the 1 -dimensional real projective space $\mathbb{R} P^{1}$ sitting inside $\mathbb{R} P^{2}$ by the standard inclusion. This finishes the proof of Lemma 3.2.5.

Fix a spin structure corresponding to an element $\sigma \in H^{1}\left(M_{2 g} ; \mathbb{Z}\right)$. Let $\Delta_{\sigma}^{+}$and $\Delta_{\sigma}^{-}$be the associated positive and negative spinor bundles. Both are 2-dimensional complex vector bundles with structure group $S U(2)$.

Remark 3.2.6. As $S U(2)$-bundles the bundles $\Delta_{\sigma}^{+}$and $\Delta_{\sigma}^{-}$do not depend on the choice of the spin structure on the manifold $M_{2 g}$. This means that for two elements $\sigma$ and $\delta \in H^{1}\left(M_{2 g} ; \mathbb{Z}_{2}\right)$ if $\Delta_{\sigma}^{+}, \Delta_{\sigma}^{-}$and $\Delta_{\delta}^{+}, \Delta_{\delta}^{-}$denote the associated positive and negative spin bundles, then as $S U(2)$-bundles $\Delta_{\sigma}^{+} \cong \Delta_{\delta}^{+}$and $\Delta_{\sigma}^{-} \cong \Delta_{\delta}^{-}$. This can be proved using an idea which is stated in [LM p. 84].

Recall that the Lie group $H=S U(2)$ acts on the manifold $M_{2 g}$ by acting trivially on the surface $\mathcal{F}_{2 g}$ and by the action of $S O(3)$ on $S(v)$. This action on the manifold $M_{2 g}$ has no fixed points and lifts canonically to the tangent bundle $T M_{2 g} \cong T \mathcal{F}_{2 g} \times{ }_{\sigma} T S(v)$. Since the Lie group $S U(2)$ is connected and simply connected it lifts to any spin bundle over the manifold $M_{2 g}$ covering the action on the tangent bundle. Hence it also lifts to the positive and negative spinor bundles $\Delta^{+}$and $\Delta^{-}$. These $S U(2)$-actions on the bundles $\Delta^{+}$and $\Delta^{-}$are lifts of the induced $S U(2)$-actions on the bundles $E_{+}=T M_{2 g} \times_{\rho_{+}} \Lambda_{+}^{2}\left(\mathbb{R}^{4}\right)$ and $E_{-}=$ $T M_{2 g} \times \rho_{-} \Lambda_{-}^{2}\left(\mathbb{R}^{4}\right)$ to a spin structure on $E_{+}$and $E_{-}$. Here $\rho_{ \pm}$denote the two non-equivalent irreducible three dimensional representations of $S O(4)$ on $\Lambda_{ \pm}^{2}\left(\mathbb{R}^{4}\right)$. These lifts from the bundles $E_{+}$and $E_{-}$to the bundles $\Delta^{+}$and $\Delta^{-}$are unique since each two lifts differ by maps $\alpha_{ \pm}: S U(2) \times P_{\text {Spin }}\left(E_{ \pm}\right) \rightarrow \mathbb{Z}_{2}$ such that $\alpha_{ \pm}(\mathrm{Id}, p)=1$ for each $p \in P_{\text {Spin }}\left(E_{ \pm}\right)$. Here we denote by $P_{\text {Spin }}\left(E_{+}\right)$the principal spin bundle of the bundle $E_{+}$and define $P_{\text {Spin }}\left(E_{-}\right)$similarly. Since the product $S U(2) \times P_{\text {Spin }}\left(E_{ \pm}\right)$ is connected, the maps $\alpha_{ \pm}$have to be the constant maps $\alpha_{ \pm}(\lambda, p)=1 \in \mathbb{Z}_{2}$ for all elements $\lambda \in S U(2)$ and $p \in P_{\text {Spin }}\left(E_{ \pm}\right)$.

We now compute the second Chern classes $c_{2}\left(\Delta^{+}\right)$and $c_{2}\left(\Delta^{-}\right)$of the bundles $\Delta^{+}$ and $\Delta^{-}$. Since we have the relations $p_{1}\left(E_{+}\right)=-4 c_{2}\left(\Delta^{+}\right)$and $p_{1}\left(E_{-}\right)=-4 c_{2}\left(\Delta^{-}\right)$ between the first Chern classes and the first Pontryagin classes, it is enough to compute the first Pontryagin classes of the bundles $E_{ \pm}$.

Proposition 3.2.7. Let $E_{+}=T M_{2 g} \times_{\rho_{+}} \Lambda_{+}^{2}\left(\mathbb{R}^{4}\right)$ and $E_{-}=T M_{2 g} \times{ }_{\rho_{-}} \Lambda_{-}^{2}\left(\mathbb{R}^{4}\right)$ be the vector bundles induced from the tangent bundle of the manifold $M_{2 g}$ by the representations $\rho_{ \pm}: S O(4) \rightarrow G L\left(\Lambda_{ \pm}^{2}\left(\mathbb{R}^{4}\right)\right)$. The first Pontryagin classes are $p_{1}\left(E_{+}\right)=4(1-2 g)$ and $p_{1}\left(E_{-}\right)=-4(1-2 g)$.

Proof. $p_{1}\left(E_{-}\right)$follows from $p_{1}\left(E_{+}\right)$by changing the orientation. Let $T \subset S O(4)$ be the standard maximal torus $T \cong S O(2) \times S O(2)$ in the compact Lie group $S O(4)$ and let $T^{\prime} \subset S O(3)$ be the maximal torus

$$
T^{\prime}=\left\{\left(\begin{array}{ccc}
1 & 0 & 0 \\
0 & \cos \theta & -\sin \theta \\
0 & \sin \theta & \cos \theta
\end{array}\right) \mid 0 \leq \theta \leq 2 \pi\right\} .
$$


We compute for the representation $\rho_{+}$restricted to the maximal torus $T$ inside $S O(4)$ that $\rho_{+}(T) \subset T^{\prime}$ and

$$
\rho_{+}\left(\begin{array}{cccc}
\cos \theta_{1} & -\sin \theta_{1} & 0 & 0 \\
\sin \theta_{1} & \cos \theta_{1} & 0 & 0 \\
0 & 0 & \cos \theta_{2} & -\sin \theta_{2} \\
0 & 0 & \sin \theta_{2} & \cos \theta_{2}
\end{array}\right)=\left(\begin{array}{cccc}
1 & 0 & 0 \\
0 & \cos \left(\theta_{1}+\theta_{2}\right) & -\sin \left(\theta_{1}+\theta_{2}\right) \\
0 & \sin \left(\theta_{1}+\theta_{2}\right) & \cos \left(\theta_{1}+\theta_{2}\right)
\end{array}\right) .
$$

Let $y \in H^{1}\left(T^{\prime} ; \mathbb{Z}\right)$ and $x_{1}, x_{2} \in H^{1}(T, \mathbb{Z})$ be the generators. Then the induced map in cohomology is given by

$$
\begin{aligned}
\rho_{+}^{*}: H^{1}\left(T^{\prime} ; \mathbb{Z}\right) & \rightarrow H^{1}(T ; \mathbb{Z}) \\
\rho_{+}^{*}(y) & =x_{1}+x_{2} .
\end{aligned}
$$

By the splitting principle we may assume that $T M_{2 g} \cong l_{1} \oplus l_{2}$ where $l_{1}$ and $l_{2}$ are $S O(2)$-bundles over the manifold $M_{2 g}$ and $x_{i}=c_{1}\left(l_{i}\right)$ for $i=1,2$. By $\mathrm{BoH}$. Theorem 10.3.b, p. 491] we obtain for the total Pontryagin classes of the vector bundle $E_{+}$

$$
\begin{aligned}
2 p\left(E_{+}\right)=1+\left(x_{1}+x_{2}\right)^{2} & =1+x_{1}^{2}+x_{2}^{2}+2 x_{1} x_{2} \\
& =1+p_{1}\left(T M_{2 g}\right)+2 e\left(T M_{2 g}\right) .
\end{aligned}
$$

where $p_{1}\left(T M_{2 g}\right)$ denotes the first Pontryagin class and $e\left(T M_{2 g}\right)$ the Euler class of the tangent bundle of the manifold $M_{2 g}$. Hence we obtain from (3.2.8) and Lemma 3.2 .9 below the equality $p_{1}\left(E_{+}\right)=2(2-2(2 g))=4(1-2 g)$. This finishes the proof Of Proposition 3.2.7.

Lemma 3.2.9. (i) $e\left(T M_{2 g}\right)=2-2(2 g)$

(ii) $p_{1}\left(T M_{2 g}\right)=0$.

Proof of Lemma 3.2.9. Let $\pi: \mathcal{F}_{2 g} \times S(v) \rightarrow \mathcal{F}_{2 g} \times_{\sigma} S(v)=M_{2 g}$ be the canonical projection. On the fourth cohomology group $H^{4}$ with integer coefficients the induced map $\pi^{*}$ is multiplication by 2 . Also we obviously obtain for the pull back bundle of the tangent bundle $T M_{2 g}$ the isomorphism $\pi^{*}\left(T M_{2 g}\right) \cong T \mathcal{F}_{2 g} \times T S(v)$. As complex line bundles we write $T \mathcal{F}_{2 g}=l_{2 g}$ and $T S(v)=l_{0}$ with the dual bundles $\bar{l}_{2 g}$ and $\bar{l}_{0}$.

For (ii) we obtain

$$
\begin{aligned}
\pi^{*}\left(p_{1}\left(T M_{2 g}\right)\right) & =2 p_{1}\left(T M_{2 g}\right) \\
& =p_{1}\left(T \mathcal{F}_{2 g} \times T S(v)\right)=-c_{2}\left(\left(T \mathcal{F}_{2 g} \times T S(v)\right) \otimes \mathbb{C}\right) \\
& =-c_{2}\left(\left(l_{2 g} \oplus \bar{l}_{2 g}\right) \times\left(l_{0} \oplus \bar{l}_{0}\right)\right) \\
& =1 \times c_{1}\left(l_{0}\right)^{2}+c_{1}\left(l_{2 g}\right)^{2} \times 1 \\
& =0 \quad \text { evaluated on } H^{4}\left(\mathcal{F}_{2 g} \times S(v) ; \mathbb{Z}\right)
\end{aligned}
$$

since the classes $c_{1}\left(l_{0}\right)^{2}$ evaluated on $H^{*}(S(v), \mathbb{Z})$ and $c_{1}\left(l_{2 g}\right)^{2}$ evaluated on $H^{*}\left(\mathcal{F}_{2 g} ; \mathbb{Z}\right)$ are zero.

For (i) we obtain

$$
\begin{aligned}
\pi^{*}\left(e\left(T M_{2 g}\right)\right) & =2 e\left(T M_{2 g}\right) \\
& =e\left(T \mathcal{F}_{2 g} \times T S(v)\right)=e\left(T \mathcal{F}_{2 g}\right) \times e(T S(v)) \\
& =(2-2(2 g)) 2 \quad \text { evaluated on } H^{4}\left(\mathcal{F}_{2 g} \times S(v) ; \mathbb{Z}\right) .
\end{aligned}
$$

This gives $e\left(T M_{2 g}\right)=2-2(2 g)$ and finishes the proof of Lemma 3.2.9. 
Corollary 3.2.10. The second Chern classes of the positive and negative spinor bundles $\Delta^{ \pm}$over the manifold $M_{2 g}$ are given by $c_{2}\left(\Delta^{+}\right)=2 g-1$ and $c_{2}\left(\Delta^{-}\right)=$ $1-2 g$.

We can now set up the equivariant Morse theory. Fix an $S U(2)$-invariant metric on the manifold $M_{2 g}$.

Remark 3.2.11. Since $H^{2}\left(M_{2 g} ; \mathbb{Q}\right)=0$ and since the bundles $\Delta^{ \pm}$have non-zero second Chern classes by Theorem 3.1 in [FU, p. 47] (see also [Pa1, Lemma 3.3, p. 346]) they cannot admit connections with stabilizer in the equivariant gauge group larger than the constant $\mathbb{Z}_{2} \subset S U(2)$ and hence they do not admit reducible connections.

By Corollary 3.2.10 if $g=0$, then the bundle $\Delta^{+}$does not admit anti-self dual connections and the bundle $\Delta^{-}$does not admit self dual connections. If $g \geq 1$ the bundle $\Delta^{+}$does not admit self dual connections and the bundle $\Delta^{-}$does not admit anti-self dual connections. We fix the following conventions: For $g=0$ we study connections on the bundle $\Delta^{-}$and for $g \geq 1$ we study connections on the bundle $\Delta^{+}$. In the case $g=0$ we define $\mathcal{B}_{S U(2)}$ to be the $S U(2)$-invariant connections on the bundle $\Delta^{-}$modulo the invariant gauge group. In the case $g \geq 1$ we define $\mathcal{B}_{S U(2)}$ to be the $S U(2)$ - invariant connections on the bundle $\Delta^{+}$modulo the invariant gauge group. Let $\mathcal{M}_{S U(2)} \subset \mathcal{B}_{S U(2)}$ denote the subspace of $S U(2)$-invariant anti-self dual connections modulo the invariant gauge group on the bundle $\Delta^{-}$(if $g=0$ ) or $\Delta^{+}$(if $g \geq 1$ ). By changing the orientation we obtain theorems on the existence of critical points of the Yang-Mills functional which are not self dual on the "opposite" bundles.

In order to use Morse or Lusternik-Schnirelman theory we would like to know the weak homotopy type of the invariant orbit space $\mathcal{B}_{S U(2)}$. Therefore in the next section we will compute the homotopy type of this space.

3.3 Computation of the weak homotopy type of the space $\mathcal{B}_{S U(2)}$. Let $f_{0} \in \mathcal{F}_{2 g}$ be a fixed point of the involution $\sigma_{1}: \mathcal{F}_{2 g} \rightarrow \mathcal{F}_{2 g}$ defined in (3.2.1) and $e_{0} \in S(v)$ the point $(1,0,0)$ of the two sphere $S(v)$. Then on the manifold $M_{2 g}=\mathcal{F}_{2 g} \times_{\sigma} S(v)$ we choose the point $\left[f_{0}, e_{0}\right] \in \mathcal{F}_{2 g} \times_{\sigma} S(v)$ as the base point.

Since the action of the Lie group $S O(3)$ on the two sphere $S(v)$ is transitive, the orbit of the $S U(2)$-action on the manifold $M_{2 g}$ through the base point $\left[f_{0}, e_{0}\right] \in M_{2 g}$ is equal to the subspace $\left\{\left[f_{0}, w\right]: w \in S(v)\right\} \cong \sigma_{2} \backslash S(v) \cong \mathbb{R} P^{2}$. The $S U(2)$-action on the manifold $M_{2 g}$ has 2 types of isotropy groups. Every point $[f, w] \in \mathcal{F}_{2 g} \times{ }_{\sigma} S(v)$ is fixed by some circle $U(1) \subset S U(2)$. If the point $f \in \mathcal{F}_{2 g}$ is not fixed by the involution $\sigma_{1}: \mathcal{F}_{2 g} \rightarrow \mathcal{F}_{2 g}$, then the isotropy group of the point $[f, w]$ for any $w \in S(v)$ is conjugate to the standard circle

$$
U(1) \cong\left\{\left(\begin{array}{cc}
\exp (i \theta) & 0 \\
0 & \exp (-i \theta)
\end{array}\right): 0 \leq \theta \leq 2 \pi\right\}
$$

in the Lie group $S U(2)$. If the point $f \in \mathcal{F}_{2 g} \times{ }_{\sigma} S(v)$ is fixed by the involution $\sigma_{1}: \mathcal{F}_{2 g} \rightarrow \mathcal{F}_{2 g}$, then the isotropy group of the point $[f, w]$ for any $w \in S(v)$ is conjugate to the group $\operatorname{Pin}(2) \subset S U(2)$ which is generated by the standard circle $U(1) \subset S U(2)$ and the element $j \in S p(1) \cong S U(2)$. The group $\operatorname{Pin}(2)$ is the double cover of the group $O(2) \subset S O(3)$.

We now compute the isotropy representations of the two possible isotropy groups $U(1)$ and $\operatorname{Pin}(2)$ in $S U(2)$ on the fibers of the bundles $\Delta^{+}$and $\Delta^{-}$over the manifold 
$M_{2 g}$. Recall that the positive spinor bundle $\Delta^{+}$is a lift of the $S O(3)$-bundle $E_{+}=P\left(T M_{2 g}\right) \times{ }_{\rho_{+}} \Lambda_{+}^{2}\left(\mathbb{R}^{4}\right)$.

Let $m=[f, w] \in \mathcal{F}_{2 g} \times_{\sigma} S(v)=M_{2 g}$ be a point with isotropy group conjugate to the standard circle $U(1) \subset S U(2)$ defined in (3.3.1). The isotropy representation of the point $m$ on the fiber $\left(T M_{2 g}\right)_{m}$ of the tangent bundle $T M_{2 g}$ of the manifold $M_{2 g}$ is conjugate to the homomorphism

$$
\begin{aligned}
\tau: U(1) & \rightarrow S O(4) \\
\exp (i \theta) & \mapsto\left(\begin{array}{cccc}
1 & 0 & 0 & 0 \\
0 & 1 & 0 & 0 \\
0 & 0 & \cos (2 \theta) & -\sin (2 \theta) \\
0 & 0 & \sin (2 \theta) & \cos (2 \theta)
\end{array}\right) .
\end{aligned}
$$

Hence the isotropy representation of the circle $U(1)$ on the fiber $\left(E_{+}\right)_{m}$ of the bundle $E_{+}$is given by the homomorphism

$$
\begin{aligned}
\rho_{+} \circ \tau: U(1) & \rightarrow S O(3) \\
\exp (i \theta) & \mapsto\left(\begin{array}{ccc}
1 & 0 & 0 \\
0 & \cos (2 \theta) & -\sin (2 \theta) \\
0 & \sin (2 \theta) & \cos (2 \theta)
\end{array}\right) .
\end{aligned}
$$

Since the bundle $\Delta^{+}$is the spinor bundle of the bundle $E_{+}$for some spin structure on the bundle $E_{+}$the isotropy representation of the point $m$ on the fiber $\left(\Delta^{+}\right)_{m}$ of the bundle $\Delta^{+}$is conjugate to the representation

$$
\begin{aligned}
U(1) & \rightarrow S U(2) \\
\exp (i \theta) & \mapsto\left(\begin{array}{cc}
\exp (i \theta) & 0 \\
0 & \exp (-i \theta)
\end{array}\right) .
\end{aligned}
$$

Since the circle $U(1)$ is connected, the sign in (3.3.3) has to be constant and equal to + . Hence the isotropy representation of the point $m=[f, w] \in M_{2 g}$ is conjugate to the standard inclusion $U(1) \hookrightarrow S U(2)$ given in (3.3.2).

Now let $m=[f, w] \in \mathcal{F}_{2 g} \times{ }_{\sigma} S(v)=M_{2 g}$ be a point with isotropy group conjugate to the group $\operatorname{Pin}(2)=\langle j, U(1)\rangle \subset S U(2)$. The isotropy representation of the group $\operatorname{Pin}(2)$ on the fiber $\left(\Delta^{+}\right)_{m}$ of the positive spin bundle is a 2-dimensional complex representation. Since restricted to the circle $U(1) \subset \operatorname{Pin}(2)$ this representation is conjugate to the standard inclusion $U(1) \subset S U(2)$ (by the same argument as above) it has to be conjugate to the unique irreducible 2-dimensional complex representation of the group $\operatorname{Pin}(2)$ on $\mathbb{C}^{2}$ which is induced from the circle $U(1) \subset$ $\operatorname{Pin}(2)$ by the 1-dimensional complex representation of weight 1 (or weight -1). This representation is just given by the standard inclusion $\operatorname{Pin}(2) \hookrightarrow S U(2)$. The same arguments apply to the isotropy representations of the negative spinor bundle.

Identifying the Lie group $S U(2)$ with the Lie group $S p(1)$ we can consider the bundles $\Delta^{+}$and $\Delta^{-}$as $S U(2)$-equivariant quaternionic line bundles. Recall that an $S U(2)$-equivariant quaternionic line bundle $\eta=\left(E \rightarrow M_{2 g}\right)$ is subordinate to a quaternionic left module $V$ if for every point $m \in M_{2 g}$ the isotropy representation of the isotropy group $S U(2)_{m}$ on the fiber $E_{m}$ of the bundle $\eta$ over the point $m \in M_{2 g}$ is contained up to isomorphism in the space $V$ viewed as an $S U(2)_{m}$-module. The previous discussion proves the following proposition: 
Proposition 3.3.4. The $S U(2)$-equivariant quaternionic line bundles $\Delta^{+}$and $\Delta^{-}$ over the manifold $M_{2 g}$ are subordinate to the non-trivial 1-dimensional quaternionic $S U(2) \cong S p(1)$-module $\mathbb{H}$ given by left multiplication of the group $S p(1)$.

Let $\mathcal{G}_{S U(2)}^{+}$denote the $S U(2)$-equivariant gauge group of the bundle $\Delta^{+}$and let $\mathcal{G}_{S U(2)}^{+, 0}$ denote the subgroup of based equivariant gauge transformations, i.e. the equivariant gauge transformations on the bundle $\Delta^{+}$which are the identity on the fiber $\left(\Delta^{+}\right)_{m}$ over the base point $m=\left[f_{0}, e_{0}\right] \in \mathcal{F}_{2 g} \times_{\sigma} S(v)=M_{2 g}$. Similarly we define the equivariant gauge group $\mathcal{G}_{S U(2)}^{-}$and the based equivariant gauge group $\mathcal{G}_{S U(2)}^{-, 0}$ of the bundle $\Delta^{-}$.

Lemma 3.3.5. We have isomorphisms

(i) $\mathcal{G}_{S U(2)}^{+} / \mathcal{G}_{S U(2)}^{+, 0} \cong \mathbb{Z}_{2}$,

(ii) $\mathcal{G}_{S U(2)}^{-} / \mathcal{G}_{S U(2)}^{-, 0} \cong \mathbb{Z}_{2}$.

Proof. For the proof of (i) let $\Delta_{m}^{+}$denote the fiber of the vector bundle $\Delta^{+}$over the base point $m=\left[f_{0}, e_{0}\right] \in M_{2 g}$. Recall that the base point $m \in M_{2 g}$ has the isotropy group $\operatorname{Pin}(2) \subset S U(2)$ since we chose the point $f_{0} \in M_{2 g}$ to be a fixed point of the involution $\sigma_{1}: \mathcal{F}_{2 g} \rightarrow \mathcal{F}_{2 g}$. Define the restriction map

$$
\begin{aligned}
\Gamma_{m}: \mathcal{G}_{S U(2)}^{+} & \rightarrow G L\left(\Delta_{m}^{+}\right) \\
\phi & \left.\mapsto \phi\right|_{m} .
\end{aligned}
$$

Since the gauge transformation $\phi \in \mathcal{G}_{S U(2)}^{+}$is $S U(2)$-equivariant, it commutes with the action of the isotropy group $\operatorname{Pin}(2)$ on the space $\Delta_{m}^{+}$and hence $\left.\phi\right|_{m}$ is a $\operatorname{Pin}(2)$-module isomorphism. We have seen above that this $\operatorname{Pin}(2)$-module $\Delta_{m}^{+}$is irreducible. Therefore by Schur's lemma it must be given by multiplication with some element $\lambda \in \mathbb{C}^{*}$, i.e. $\left.\phi\right|_{m}=\lambda \mathrm{Id}: \mathbb{C}^{2} \rightarrow \mathbb{C}^{2}$. But the bundle $\Delta^{+}$has structure group $S U(2)$ and by definition the gauge transformation $\phi$ is an automorphism of the $S U(2)$-bundle $\Delta^{+}$and hence $\left.\phi\right|_{m} \in S U(2)$. This implies $\lambda \in \mathbb{Z}_{2}$. Hence the restriction map $\Gamma_{m}$ takes values in $\mathbb{Z}_{2} \subset S U(2)$ and is obviously surjective. Since the kernel of the restriction map $\Gamma_{m}$ is by definition the group $\mathcal{G}_{S U(2)}^{+, 0}$ we have proved (i). Since the proof of (ii) is the same, we have finished the proof of Lemma 3.3.5.

Let $\mathcal{B}_{S U(2)}^{0}$ denote the orbit space of $S U(2)$-invariant connections on the bundle $\Delta^{+}$if $g \geq 1$ or $\Delta^{-}$if $g=0$ modulo the action of the based invariant gauge group $\mathcal{G}_{S U(2)}^{+, 0}$ or $\mathcal{G}_{S U(2)}^{-, 0}$ respectively. Let $\pi: \mathcal{B}_{S U(2)}^{+, 0} \rightarrow \mathcal{B}_{S U(2)}^{+}$be the canonical projection. Since the bundles $\Delta^{ \pm}$do not admit a reducible connection (see Remark 3.2.11), the map $\pi$ is a fibration with fiber $\left(\mathcal{G}_{S U(2)}^{ \pm} / \mathcal{G}_{S U(2)}^{ \pm, 0}\right) / \mathbb{Z}_{2}$. By Lemma 3.3.5 we have the isomorphism $\mathcal{G}_{S U(2)}^{ \pm} / \mathcal{G}_{S U(2)}^{ \pm, 0} \cong \mathbb{Z}_{2}$ and hence the projection map $\pi$ is in fact a homeomorphism. This proves

Proposition 3.3.6. The natural projection map $\pi: \mathcal{B}_{S U(2)}^{0} \rightarrow \mathcal{B}_{S U(2)}$ is a homeomorphism.

In the remainder of this section we compute the weak homotopy type of the based invariant orbit space $\mathcal{B}_{S U(2)}$ Using Theorem 2.3.1. 
Theorem 3.3.7. There is a weak homotopy equivalence

$$
\mathcal{B}_{S U(2)}^{0} \simeq \prod_{2 g} S^{1}
$$

Proof. Let $\mathbb{H}$ be the 1-dimensional quaternionic representation of $S U(2) \cong S p(1)$ given by left multiplication. By Proposition 3.3.4 the $S U(2)$-equivariant bundles $\Delta^{ \pm}$are subordinate to this representation. Hence Theorem 2.3.1 gives a weak homotopy equivalence

$$
\mathcal{B}_{S U(2)}^{0} \simeq \operatorname{Map}_{S U(2)}^{0}\left(M_{2 g}, G_{1}\left(\mathbb{H}^{\infty}\right)\right)^{ \pm} .
$$

Here we have chosen the point $\left[f_{0}, e_{o}\right] \in \mathcal{F}_{2 g} \times_{\sigma} S(v)$ defined before as the base point and a base point $* \in G_{1}\left(\mathbb{H}^{\infty}\right)$ according to Theorem 2.2.2. Both points are fixed by the group $\operatorname{Pin}(2) \subset S U(2)$. The superscript \pm on the right hand side of (3.3.8) denotes the components of maps which classify the $S U(2)$-equivariant positive spinor bundles $\Delta^{ \pm}$over the manifold $M_{2 g}=\mathcal{F}_{2 g} \times{ }_{\sigma} S(v)$.

One checks that any line $*=\left[\left(x_{0}, x_{1}, \ldots\right)\right]$ in the infinite dimensional Grassmannian $G_{1}\left(\mathbb{H}^{\infty}\right)$ which is fixed by the group $\operatorname{Pin}(2) \subset S U(2)$ is already fixed by the whole group $S U(2)$ and hence is a fixed point of the $S U(2)$-action on the space $G_{1}\left(\mathbb{H}^{\infty}\right)$. Hence any map $f: M_{2 g} \rightarrow G_{1}\left(\mathbb{H}^{\infty}\right)$ which is base point preserving maps the whole orbit through the base point $\left[f_{0}, e_{0}\right]$ to the base point $* \in G_{1}\left(\mathbb{H}^{\infty}\right)$, i.e. $f\left(\left[f_{0}, x\right]\right)=*$ for any $x \in S(v)$. This implies the homeomorphisms

$$
\begin{aligned}
& \operatorname{Map}_{S U(2)}^{0}\left(\mathcal{F}_{2 g} \times_{\sigma} S(v), G_{1}\left(\mathbb{H}^{\infty}\right)\right) \\
& \quad \cong \operatorname{Map}_{S U(2)}^{0}\left(\frac{\mathcal{F}_{2 g} \times_{\sigma} S(v)}{\left\{f_{0}\right\} \times_{\sigma} S(v)}, G_{1}\left(\mathbb{H}^{\infty}\right)\right) .
\end{aligned}
$$

Let $S(v)_{+}=S(v) \cup\{+\}$ denote the two sphere $S(v)$ with an additional disjoint base point $\{+\}$. We choose the point $f_{0} \in \mathcal{F}_{2 g}$ as the base point in the Riemann surface $\mathcal{F}_{2 g}$ and define the smash product

$$
\mathcal{F}_{2 g} \wedge S(v)_{+}=\frac{\mathcal{F}_{2 g} \times S(v)_{+}}{\mathcal{F}_{2 g} \times\{+\} \cup\left\{f_{0}\right\} \times S(v)_{+}} .
$$

We extend the involution $\sigma_{2}: S(v) \rightarrow S(v)$ given by the reflection through the origin to the space $S(v)$ + by letting it act trivially on the additional base point. The involution $\sigma: \mathcal{F}_{2 g} \times S(v) \rightarrow \mathcal{F}_{2 g} \times S(v)$ given by $\sigma(v, w)=\left(\sigma_{1}(v), \sigma_{2}(w)\right)$ extends to the space $\mathcal{F}_{2 g} \times S(v)_{+}$and it preserves the subspace $\mathcal{F}_{2 g} \times\{+\} \cup\left\{f_{0}\right\} \times S(v)_{+} \subset$ $\mathcal{F}_{2 g} \times S(v)_{+}$. Hence we obtain an induced involution $\sigma: \mathcal{F}_{2 g} \wedge S(v)_{+} \rightarrow \mathcal{F}_{2 g} \wedge S(v)_{+}$. Let $\mathcal{F}_{2 g} \wedge_{\sigma} S(v)_{+}$denote the quotient.

We extend the $S U(2)$-action on the two sphere $S(v)$ to the space $S(v)_{+}$by letting it act trivially on the additional base point + . This induces an action on the smash $\mathcal{F}_{2 g} \wedge S(v)_{+}$which commutes with the action of the involution $\sigma$. Hence it induces an $S U(2)$-action on the quotient $\mathcal{F}_{2 g} \wedge_{\sigma} S(v)_{+}$. The canonical mapping

$$
\begin{gathered}
\varepsilon: \quad \frac{\mathcal{F}_{2 g} \times_{\sigma} S(v)}{\left\{f_{0}\right\} \times_{\sigma} S(v)} \longrightarrow \mathcal{F}_{2 g} \wedge_{\sigma} S(v)_{+} \\
\varepsilon([x, y])=[x, y]
\end{gathered}
$$

is equivariant with respect to the $S U(2)$-action and induces an $S U(2)$-equivariant homeomorphism. Together with (3.3.9) this gives a homeomorphism

$$
\operatorname{Map}_{S U(2)}^{0}\left(\mathcal{F}_{2 g} \times_{\sigma} S(v) ; G_{1}\left(\mathbb{H}^{\infty}\right)\right) \cong \operatorname{Map}_{S U(2)}^{0}\left(\mathcal{F}_{2 g} \wedge_{\sigma} S(v)_{+}, G_{1}\left(\mathbb{H}^{\infty}\right)\right) .
$$


A map $f: \mathcal{F}_{2 g} \wedge_{\sigma} S(v)_{+} \rightarrow G_{1}\left(\mathbb{H}^{\infty}\right)$ is given by a map $\tilde{f}: \mathcal{F}_{2 g} \wedge S(v)_{+} \rightarrow G_{1}\left(\mathbb{H}^{\infty}\right)$ which is invariant under the action by the involution $\sigma$. (The space $G_{1}\left(\mathbb{H}^{\infty}\right)$ is given the trivial $\sigma$-action). The adjoint map $\tilde{f}^{\text {ad }}: \mathcal{F}_{2 g} \rightarrow \operatorname{Map}^{0}\left(S(v)_{+}, G_{1}\left(\mathbb{H}^{\infty}\right)\right)$ is then invariant under a $\mathbb{Z}_{2}$-action where the group $\mathbb{Z}_{2}$ acts on the Riemann surface by the action of the involution $\sigma_{1}$ and on the mapping space $\operatorname{Map}^{0}\left(S(v)_{+}, G_{1}\left(\mathbb{H}^{\infty}\right)\right)$ by the induced action on maps given by the $\sigma_{1}$-action on the space $S(v)_{+}$and the trivial action on the Grassmannian $G_{1}\left(\mathbb{H}^{\infty}\right)$. Since the $S U(2)$-and $\sigma_{2}$-actions on the space $S(v)_{+}$commute, we obtain the homeomorphism

$$
\begin{aligned}
& \operatorname{Map}_{S U(2)}^{0}\left(\mathcal{F}_{2 g} \times_{\sigma} S(v), G_{1}\left(\mathbb{H}^{\infty}\right)\right. \\
& \quad \cong \operatorname{Map}_{\mathbb{Z}_{2}}^{0}\left(\mathcal{F}_{2 g}, \operatorname{Map}_{S U(2)}^{0}\left(S(v)_{+}, G_{1}\left(\mathbb{H}^{\infty}\right)\right)\right) .
\end{aligned}
$$

We now analyze the mapping space $\operatorname{Map}_{S U(2)}^{0}\left(S(v)_{+}, G_{1}\left(\mathbb{H}^{\infty}\right)\right)$ together with the above $\mathbb{Z}_{2}$-action. We first need to prove

Lemma 3.3.11. There is a homeomorphism $G_{1}\left(\mathbb{H}^{\infty}\right)^{U(1)} \cong G_{1}\left(\mathbb{C}^{\infty}\right)$.

Proof of Lemma 3.3.11. Let $\tau: G_{1}\left(\mathbb{C}^{\infty}\right) \hookrightarrow G_{1}\left(\mathbb{H}^{\infty}\right)$ be the map induced by the natural inclusion $\mathbb{C} \hookrightarrow \mathbb{H}$. Recall that we view the space $\mathbb{H}^{\infty}$ as a right quaternionic vector space endowed with the $U(1)$-action given by left multiplication of weight $p$. Hence the image $\tau\left(G_{1}\left(\mathbb{C}^{\infty}\right)\right)$ lies in the fixed point set $G_{1}\left(\mathbb{H}^{\infty}\right)^{U(1)}$ of the induced circle action on the Grassmannian $G_{1}\left(\mathbb{H}^{\infty}\right)$ and the map $\tau$ induces a continuous map $\tilde{\tau}: G_{1}\left(\mathbb{C}^{\infty}\right) \hookrightarrow G_{1}\left(\mathbb{H}^{\infty}\right)^{U(1)}$. Let $w=\left[x_{0}, x_{1}, \ldots\right]$ be a quaternionic line in $\mathbb{H}^{\infty}$ fixed under the circle action. Without loss of generality we may assume $x_{0}=1$. Given $\lambda \in U(1)$ there is an element $\alpha(\lambda) \in S p(1)$ such that $\lambda^{p} x_{i}=x_{i} \alpha(\lambda)$ for all $i=0,1, \ldots$ For $i=0$ this gives $\alpha(\lambda)=\lambda^{p}$. Hence $\lambda^{p} x_{i}=x_{i} \lambda^{p}$ for all $i=0,1, \ldots$ and hence $x_{i}$ lies in the centralizer of $U(1)$ in the quaternions $\mathbb{H}$ which is equal to $\mathbb{C}$. Hence $x_{i} \in \mathbb{C}$ for all $i=0,1, \ldots$ and the element $w=\left[x_{0}, x_{1}, \ldots\right]$ lies in the image of the map $\tilde{\tau}: G_{1}\left(\mathbb{C}^{\infty}\right) \rightarrow G_{1}\left(\mathbb{H}^{\infty}\right)^{U(1)}$. The map $\tilde{\tau}$ induces a homeomorphism $G_{1}\left(\mathbb{C}^{\infty}\right) \cong G_{1}\left(\mathbb{H}^{\infty}\right)^{U(1)}$. This finishes the proof of Lemma 3.3.11.

Lemma 3.3.12. There is a $\mathbb{Z}_{2}$-equivariant homeomorphism

$$
\operatorname{Map}_{S U(2)}^{0}\left(S(v)_{+}, G_{1}\left(\mathbb{H}^{\infty}\right)\right) \cong G_{1}\left(\mathbb{C}^{\infty}\right)
$$

where the group $\mathbb{Z}_{2}$ acts on the infinite dimensional Grassmannian $G_{1}\left(\mathbb{C}^{\infty}\right)=\mathbb{C} P^{\infty}$ by complex conjugation on coordinates.

Proof. The inclusion $S(v) \hookrightarrow S(v)_{+}$induces a homeomorphism

$$
\operatorname{Map}_{S U(2)}^{0}\left(S(v)_{+}, G_{1}\left(\mathbb{H}^{\infty}\right)\right) \cong \operatorname{Map}_{S U(2)}\left(S(v), G_{1}\left(\mathbb{H}^{\infty}\right)\right) .
$$

Let $N \in S(v)$ denote the north pole. The evaluation map

$$
\begin{aligned}
\operatorname{Map}_{S U(2)}\left(S(v), G_{1}\left(\mathbb{H}^{\infty}\right)\right) & \rightarrow G_{1}\left(\mathbb{H}^{\infty}\right)^{U(1)} \\
f & \mapsto f(N)
\end{aligned}
$$

induces an $S U(2)$-equivariant homeomorphism. Here we identify the two sphere $S(v)$ with the homogeneous space $S U(2) / U(1)$. By Lemma 3.3.11 there is a homeomorphism $G_{1}\left(\mathbb{C}^{\infty}\right) \cong G_{1}\left(\mathbb{H}^{\infty}\right)^{U(1)}$.

We now compute the $\mathbb{Z}_{2}$-action on the infinite dimensional complex Grassmannian $G_{1}\left(\mathbb{C}^{\infty}\right)$ induced by the homeomorphism in (3.3.13). Let $\tau$ be a generator of $\mathbb{Z}_{2}$. For any map $f \in \operatorname{Map}_{S U(2)}\left(S(v), G_{1}\left(\mathbb{H}^{\infty}\right)\right)$ the map $\tau \cdot f$ is given by $\tau \cdot f(w)=f\left(\sigma_{2}(w)\right)$. Hence $\tau \cdot f(N)=f\left(\sigma_{2}(N)\right)=f(-N)=f(S)$. Here we 
denote by $S \in S(v)$ the south pole. Let $A \in S U(2)$ be an element such that $A(N)=S$. Since the map $f$ is equivariant with respect to the $S U(2)$-action, we obtain $\tau \cdot f(N)=f\left(\sigma_{2}(N)\right)=f(S)=f(A(N))=A(f(N))$. If $B$ is another element in $S U(2)$ such that $B(N)=S$, then $B=C \cdot A$ for some element $C \in U(1) \subset S U(2)$. Here $U(1) \subset S U(2)$ is the stabilizer of the $S U(2)$-action on $S(v)$ at the north pole $N$. But since $f(N)$ lies in the fixed point set of the $U(1)$-action on the infinite dimensional Grassmannian $G_{1}\left(\mathbb{H}^{\infty}\right)$, we obtain $B(f(N))=A(f(N))$. Hence the $\mathbb{Z}_{2}$-action on the space $\left.G_{1}\left(\mathbb{H}^{\infty}\right)\right)^{U(1)}$ is given by left multiplication by any element $A \in S U(2)$ such that $A(N)=S$.

We now identify $S U(2)$ with $S p(1)$ and the two sphere $S(v)$ with the purely imaginary quaternions of norm 1 . Then the $S U(2)$-action on the 2 -sphere $S(v)$ is given by conjugation on quaternions. The north pole $N$ is identified with the quaternion $k$. Since $j k \bar{j}=-j k j=-k$, we can choose the element $A \in S U(2)$ to be conjugation by the quaternion $j \in S p(1)$. Hence the $\mathbb{Z}_{2}$-action on the space $G_{1}\left(\mathbb{H}^{\infty}\right)^{U(1)}$ induced by the homeomorphism (3.3.13) is given by left multiplication by the quaternion $j$. Let $\left[x_{0}, x_{1}, \ldots\right]$ be a line in the Grassmannian $G_{1}\left(\mathbb{H}^{\infty}\right)$ invariant under the $U(1)$-action. By Lemma 3.3.11 we may assume that the coordinates $x_{i} \in \mathbb{H}$ are all complex, i.e. $x_{i} \in \mathbb{C}$ for all $i$. Hence

$$
j \cdot\left[x_{0}, x_{1}, \ldots\right]=\left[j x_{0}, j x_{1}, \ldots\right]=\left[\bar{x}_{0} j, \bar{x}_{1} j, \ldots\right]=\left[\bar{x}_{0}, \bar{x}_{1}, \ldots\right]
$$

and therefore the $\mathbb{Z}_{2}$-action on the space $G_{1}\left(\mathbb{H}^{\infty}\right)^{U(1)} \cong G_{1}\left(\mathbb{C}^{\infty}\right)$ is given by complex conjugation. This finishes the proof of Lemma 3.3.12.

Hence we have proved

Proposition 3.3.14. There is a canonical homeomorphism

$$
\operatorname{Map}_{S U(2)}^{0}\left(\mathcal{F}_{2 g} \times{ }_{\sigma} S(v), G_{1}\left(\mathbb{H}^{\infty}\right)\right) \cong \operatorname{Map}_{\mathbb{Z}_{2}}^{0}\left(\mathcal{F}_{2 g}, G_{1}\left(\mathbb{C}^{\infty}\right)\right)
$$

where the $\mathbb{Z}_{2}$-action on the Riemann surface $\mathcal{F}_{2 g}$ is given by the involution $\sigma_{1}$ defined in (3.2.1) and on the infinite dimensional Grassmannian $G_{1}\left(\mathbb{C}^{\infty}\right)$ by complex conjugation.

We now compute the homotopy type of the $\mathbb{Z}_{2}$-equivariant mapping space $\operatorname{Map}_{\mathbb{Z}_{2}}^{0}\left(\mathcal{F}_{2 g}, G_{1}\left(\mathbb{C}^{\infty}\right)\right)$. The inclusion of the "gluing circle" $S^{1}$ into the Riemann surface $\mathcal{F}_{2 g}=\left(\mathcal{F}_{g}-D^{2}\right) \cup_{S^{1}}\left(\mathcal{F}_{g}-D^{2}\right)$ gives a $\mathbb{Z}_{2}$-equivariant cofiber sequence

$$
S^{1} \hookrightarrow \mathcal{F}_{2 g} \rightarrow \mathbb{Z}_{2}^{+} \wedge \mathcal{F}_{g}
$$

where the non-trivial element $-1 \in \mathbb{Z}_{2}$ acts on the surface $\mathcal{F}_{2 g}$ by reflection on the symmetry plane. Applying the equivariant mapping functor $\operatorname{Map}_{\mathbb{Z}_{2}}^{0}\left(\ldots, G_{1}\left(\mathbb{C}^{\infty}\right)\right)$ to (3.3.15) gives a fibration

$$
\begin{aligned}
\operatorname{Map}_{\mathbb{Z}_{2}}^{0}\left(S^{1}, G_{1}\left(\mathbb{C}^{\infty}\right)\right) & \leftarrow \operatorname{Map}_{\mathbb{Z}_{2}}^{0}\left(\mathcal{F}_{2 g}, G_{1}\left(\mathbb{C}^{\infty}\right)\right) \\
& \leftarrow \operatorname{Map}_{\mathbb{Z}_{2}}^{0}\left(\mathbb{Z}_{2}^{+} \wedge \mathcal{F}_{g}, G_{1}\left(\mathbb{C}^{\infty}\right)\right)
\end{aligned}
$$

Since the group $\mathbb{Z}_{2}$ acts trivially on the circle $S^{1}$, the base space of the fibration (3.3.16) is homeomorphic to the space $\Omega\left(G_{1}\left(\mathbb{C}^{\infty}\right)^{\mathbb{Z}_{2}}\right)$. Similarly as in Lemma 3.3 .11 one computes that the inclusion $G_{1}\left(\mathbb{R}^{\infty}\right) \hookrightarrow G_{1}\left(\mathbb{C}^{\infty}\right)$ induced by the canonical

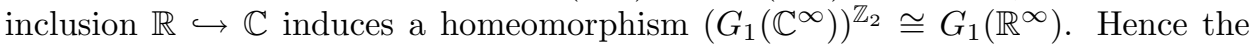
base space of fibration (3.3.16) is homotopy equivalent to the 2 -point space $\mathbb{Z}_{2}$, the 
total space is homotopy equivalent to the disjoint union of two copies of the fiber and we obtain the homotopy equivalence

$$
\operatorname{Map}_{\mathbb{Z}_{2}}^{0}\left(\mathcal{F}_{2 g}, G_{1}\left(\mathbb{C}^{\infty}\right)\right) \simeq \bigsqcup_{2 \text { copies }} \operatorname{Map}_{\mathbb{Z}_{2}}^{0}\left(\mathbb{Z}_{2}^{+} \wedge \mathcal{F}_{g}, G_{1}\left(\mathbb{C}^{\infty}\right)\right)
$$

There is a natural homeomorphism

$$
\operatorname{Map}_{\mathbb{Z}_{2}}^{0}\left(\mathbb{Z}_{2}^{+} \wedge \mathcal{F}_{g}, G_{1}\left(\mathbb{C}^{\infty}\right)\right) \cong \operatorname{Map}^{0}\left(\mathcal{F}_{g}, G_{1}\left(\mathbb{C}^{\infty}\right)\right)
$$

Since $G_{1}\left(\mathbb{C}^{\infty}\right)=B U(1)$, there is a canonical homotopy equivalence

$$
\operatorname{Map}^{0}\left(\mathcal{F}_{g}, G_{1}\left(\mathbb{C}^{\infty}\right)\right) \simeq \mathbb{Z} \times \prod_{2 g} S^{1} .
$$

Here we have switched from the notation $U(1)$ for the circle as a group to $S^{1}$ for the circle as a topological space since a mapping space is not necessarily a group. Therefore we obtain the homotopy equivalence

$$
\operatorname{Map}_{\mathbb{Z}_{2}}^{0}\left(\mathcal{F}_{2 g}, G_{1}\left(\mathbb{C}^{\infty}\right)\right) \simeq \bigsqcup_{2 \text { copies }}\left(\mathbb{Z} \times \prod_{2 g} S^{1}\right)
$$

Hence, using Proposition 3.3.14, we obtain a homotopy equivalence

$$
\operatorname{Map}_{S U(2)}^{0}\left(\mathcal{F}_{2 g} \times{ }_{\sigma} S(v), G_{1}\left(\mathbb{H}^{\infty}\right)\right) \simeq \bigsqcup_{2 \text { copies }}\left(\mathbb{Z} \times \prod_{2 g} S^{1}\right)
$$

and each component of the mapping space in (3.3.19) is homotopy equivalent to the $2 g$-fold product of the circle $S^{1}$. Together with (3.3.8) this finishes the proof of Theorem 3.3.7.

3.4 Proof of the main theorem. Recall that we defined the space $\mathcal{M}_{S U(2)} \subset$ $\mathcal{B}_{S U(2)}$ to be the space of invariant anti-self dual connections modulo the invariant gauge group on the bundle $\Delta^{-}$if $g=0$ and on the bundle $\Delta^{+}$if $g \geq 1$. Since by remark 3.2.11 the bundles $\Delta^{+}$and $\Delta^{-}$do not admit reducible connections; the invariant moduli space $\mathcal{M}_{S U(2)}$ consists of irreducible connections. By proposition 3.1 (p. 446) in [Ba] (see also Theorem 4.6 (p. 248) and section 5 in [Cho]) for a set of $S U(2)$-invariant $C^{q}$-metrics $(q \geq 1)$ on the manifold $M_{2 g}$, open and dense in the set of all invariant metrics, the invariant moduli space of anti-self dual connections $\mathcal{M}_{S U(2)}$ is a smooth (possibly empty) manifold of a dimension which can be computed using the Atiyah-Segal-Singer fixed point formula.

Remark 3.4.1. Proposition 4.1 in $[\mathrm{Ba}]$ is stated for the case $H=U(1)$ but the proof carries over word for word to the case of any compact Lie group.

Remark 3.4.2. The following argument (given to me by J. Råde) shows that Uhlenbeck's generic metrics theorem ([FU, Theorem, 3.17, p. 59]) and its equivariant versions in $\mathrm{Ba}$ and $\mathrm{ChO}$ are also true in our Morrey space completions. Any anti-self dual connection $A \in \mathcal{M}_{S U(2)}$ (completed in the $L_{3}^{1,2}$-norm) is smooth since it is a Yang-Mills connection. So we get the same moduli spaces no matter whether we work with Morrey or Sobolev spaces. In a neighborhood of an anti-self dual connection $A$ the moduli space $\mathcal{M}_{S U(2)}$ is a manifold if and only if the operator $D_{A}^{+}: \Omega_{+}^{2}(\operatorname{ad}(\eta)) \rightarrow \Omega^{1}(\operatorname{ad}(\eta))$ has trivial null space. Here $\operatorname{ad}(\eta)$ is the vector bundle associated to the given $S U(2)$-bundle $\eta$ via the adjoint representation of $S U(2) . D_{A}^{+}$is an elliptic operator with smooth coefficients so anything in the null space is smooth. Hence the condition that the operator $D_{A}^{+}$has trivial null space is independent of the choice of the function spaces. 
We now compute the formal dimension of the moduli space of invariant antiself dual connections. Assume that the moduli space $\mathcal{M}_{S U(2)}$ is not empty. By standard gauge theory and an argument in [AHS, p. 444 and 445] it follows that for a generic invariant metric the equivariant index of the Dirac operator

$$
\not D_{E}^{-}: \Gamma\left(\Delta^{-} \otimes \Delta^{+} \otimes(\operatorname{ad}(\eta) \otimes \mathbb{C})\right) \rightarrow \Gamma\left(\Delta^{+} \otimes \Delta^{+} \otimes(\operatorname{ad}(\eta) \otimes \mathbb{C})\right)
$$

is an actual representation. Here $E=\Delta^{+} \otimes(\operatorname{ad}(\eta) \otimes \mathbb{C})$ denotes the coefficient bundle. Also by standard equivariant gauge theory for a generic invariant metric the dimension of the moduli space $\mathcal{M}_{S U(2)}$ is equal to the dimension of the trivial representation contained in the representation ind $\left(\not D_{E}^{-}\right)$.

Let

$$
h=\left(\begin{array}{cc}
e^{i \theta} & 0 \\
0 & e^{-i \theta}
\end{array}\right)
$$

be an element in the standard maximal torus $U(1) \subset S U(2)$. If the angle $\theta$ is irrational, then the closure of the cyclic group generated by the element $h$ in $S U(2)$ is just the torus $U(1)$ defined in (3.3.2). The action of the element $h$ on the two sphere $S(v)$ fixes the point $e_{0}^{+}=(1,0,0)$ and $e_{0}^{-}=(-1,0,0)$. Let $M_{2 g}^{h}$ denote the fixed point set of the element $h \in S U(2)$ acting on the manifold $M_{2 g}$. Then $M_{2 g}^{h}=\mathcal{F}_{2 g} \times_{\sigma}\left\{e_{0}^{+}, e_{0}^{-}\right\} \cong \mathcal{F}_{2 g}$ where the isomorphism is induced by the inclusion $\mathcal{F}_{2 g} \hookrightarrow M_{2 g}, f \mapsto\left[f, e_{0}^{+}\right]$.

The tangent bundle $T M_{2 g}$ of the manifold $M_{2 g}$ restricted to the fixed point set $M_{2 g}^{h}$ splits $U(1)=\overline{\langle h\rangle}$-equivariantly as

$$
T M_{2 g} \cong T \mathcal{F}_{2 g}^{0} \oplus N(2 \theta)
$$

where $T \mathcal{F}_{2 g}^{0}$ denotes the real tangent bundle of the surface $\mathcal{F}_{2 g}$ together with the trivial $U(1)$-action and $N(2 \theta)$ denotes the real two dimensional trivial bundle. Here the generator $h=e^{i \theta}$ of the circle $U(1)$ acts on the bundle $N(2 \theta)$ by the matrix

$$
\left(\begin{array}{cc}
\cos (2 \theta) & -\sin (2 \theta) \\
\sin (2 \theta) & \sin (2 \theta)
\end{array}\right)
$$

The bundle $N(2 \theta)$ is the equivariant normal bundle of the fixed point set $M_{2 g}^{h} \cong \mathcal{F}_{2 g}$ inside the 4-manifold $M_{2 g}$. Both bundles in (3.4.4) are the underlying real bundles of complex line bundles on the surface $\mathcal{F}_{2 g}$.

By the $G$-Index Theorem ( $\mathrm{AS}, 5.4$, p. 572] we obtain

$$
\operatorname{ind}_{h}\left(\not D_{E}^{-}\right)= \pm \operatorname{ch}_{h}\left(\Delta^{+}\right) \operatorname{ch}_{h}(\operatorname{ad}(\eta) \otimes \mathbb{C})\left(-\hat{A}_{2 \theta}(N(2 \theta))\right) \hat{A}\left(\mathcal{F}_{2 g}\right)\left[\mathcal{F}_{2 g}\right]
$$

Here $\operatorname{ind}_{h}\left(\not D_{E}^{-}\right)$denotes the index of the operator $\not D_{E}^{-}$evaluated on the element $h \in U(1) \subset S U(2), \mathrm{ch}_{h}$ the equivariant Chern character, $\hat{A}\left(\mathcal{F}_{2 g}\right)$ the $\hat{A}$-genus of the surface $\mathcal{F}_{2 g}$ and $\hat{A}_{2 \theta}(N(2 \theta))$ a certain characteristic class to be computed later. 
Let $x \in H^{2}\left(\mathcal{F}_{2 g}, \mathbb{Q}\right)$ be the first Chern class of the complex tangent bundle of the Riemann surface $\mathcal{F}_{2 g}$. We compute:

$$
\begin{aligned}
\hat{A}\left(\mathcal{F}_{2 g}\right) & =\frac{x}{e^{\frac{x}{2}}-e^{\frac{-x}{2}}}, \\
\hat{A}(N(2 \theta)) & =\frac{e^{\frac{1}{2}(2 i \theta)}}{e^{2 i \theta}-1}=\frac{1}{e^{i \theta}-e^{-i \theta}}, \\
\operatorname{ch}_{h}\left(\Delta^{ \pm}\right) & =e^{\frac{-x}{2}} e^{\mp i \theta}+e^{\frac{x}{2}} e^{ \pm i \theta}, \\
\operatorname{ch}_{h}\left(\operatorname{ad}\left(\Delta^{ \pm}\right) \otimes \mathbb{C}\right) & =e^{-x} e^{\mp 2 i \theta}+1+e^{x} e^{ \pm 2 i \theta} .
\end{aligned}
$$

Hence for $g=0$ and $\eta=\Delta^{-}$we obtain

$$
\begin{aligned}
\operatorname{ind}_{h}\left(\not D_{E}^{-}\right) & =-\frac{\left(e^{-\frac{x}{2}} e^{-i \theta}+e^{\frac{x}{2}} e^{i \theta}\right)\left(e^{-x} e^{2 i \theta}+1+e^{x} e^{-2 i \theta}\right) x}{\left(e^{i \theta}-e^{-i \theta}\right)\left(e^{\frac{x}{2}}-e^{-\frac{x}{2}}\right)} \quad\left[S^{2}\right] \\
& =e^{2 i \theta}+3+e^{-2 i \theta}
\end{aligned}
$$

For $g \geq 1$ and $\Delta^{+}$we obtain

$$
\begin{aligned}
\operatorname{ind}_{h}\left(D_{E}^{-}\right) & =-\frac{\left(e^{-\frac{x}{2}} e^{-i \theta}+e^{\frac{x}{2}} e^{i \theta}\right)\left(e^{-x} e^{-2 i \theta}+1+e^{x} e^{2 i \theta}\right) x}{\left(e^{i \theta}-e^{-i \theta}\right)\left(e^{\frac{x}{2}}-e^{-\frac{x}{2}}\right)} \quad\left[\mathcal{F}_{2 g}\right] \\
& =(2 g-1)\left(3 e^{2 i \theta}+5+3 e^{-2 i \theta}\right) .
\end{aligned}
$$

Remark 3.4.8. Careful investigation shows that the sign in formula (3.4.5) turns out to be +1 .

Hence the dimension of the trivial representation $V_{2 g} \subset \operatorname{ind}\left(\not D_{E}^{-}\right)$is

$$
\operatorname{dim}\left(V_{2 g}\right)= \begin{cases}2, & \text { if } g=0 \\ 2(2 g-1), & \text { if } g \geq 1\end{cases}
$$

This gives

Theorem 3.4.9. If $g=0$, then either the moduli space of invariant anti-self dual connections $\mathcal{M}_{S U(2)}$ of the bundle $\Delta^{-}$is empty or, for a generic $S U(2)$-invariant metric a manifold of dimension 2. If $g \geq 1$, then either the moduli space of invariant anti-self dual connections $\mathcal{M}_{S U(2)}$ on the bundle $\Delta^{+}$is empty or, for a generic $S U(2)$-invariant metric a manifold of dimension $2(2 g-1)$.

We are now able to prove the main theorem of this section:

Theorem 3.4.10. Fix $g=0,1,2, \ldots$ and let $\Delta^{+}$and $\Delta^{-}$be the positive and negative $S U(2)$-equivariant spinor bundles over the 4-manifold $M_{2 g}=\mathcal{F}_{2 g} \times_{\sigma} S(v)$ of second Chern class $c_{2}\left(\Delta^{+}\right)=2 g-1$ and $c_{2}\left(\Delta^{-}\right)=1-2 g$. Fix an $S U(2)$-invariant metric.

(i) The Yang-Mills functional restricted to the invariant orbit space $\mathcal{Y} M: \mathcal{B}_{S U(2)}$ $\rightarrow \mathbb{R}$ has at least $2 g+1$ critical points on each of the bundles $\Delta^{+}$and $\Delta^{-}$.

(ii) In the case $g=0$, for a generic $S U(2)$-invariant metric, the critical point on the bundle $\Delta^{+}$cannot be self dual and on the bundle $\Delta^{-}$it cannot be anti-self dual. In the case $g \geq 2$ for a generic $S U(2)$-invariant metric at least one of the critical points on the bundle $\Delta^{+}$cannot be anti-self dual and at least one of the critical points on the bundle $\Delta^{-}$cannot be self dual. 
By Palais' principle of symmetric criticality in $\mathrm{Pal2}$ ] each of the critical points in (i) is a critical point of the Yang-Mills functional on the non-equivariant orbit space $\mathcal{Y} M: \mathcal{B}^{+} \rightarrow \mathbb{R}$, i.e. they are Yang-Mills connections.

Proof. Since the Yang-Mills functional is bounded from below and the invariant orbit space $\mathcal{B}_{S U(2)}$ is a complete Riemannian manifold, we can apply the classical theorem on Lusternik-Schnirelman theory by Palais ([Pal1, Theorem 7.2, p. 131]). For a topological space $X$ let $\operatorname{cat}(X)$ be the category of $X$, that is the least integer $n$ so that $\mathrm{X}$ can be covered by $n$ closed contractible subsets of $X$. Also define $\operatorname{cl}(X)$, the cuplength of the space $X$, to be the largest integer $k$ such that there exists a ring $R$ and cohomology classes $\alpha_{1}, \ldots, \alpha_{k-1} \in H^{*}(X, R)$ with positive dimension such that their cup product does not vanish. One always has the inequality $\operatorname{cat}(X) \geq$ $\operatorname{cl}(X)$.

Palais' theorem says that the Yang-Mills functional on the space $\mathcal{B}_{S U(2)}$ has at least $\operatorname{cat}\left(\mathcal{B}_{S U(2)}\right)$ critical points. Theorem 3.3.7, Proposition 3.3.6 and the inequality $\operatorname{cat}(X) \geq \operatorname{cl}(X)$ imply that the Yang-Mills functional on the space $\mathcal{B}_{S U(2)}$ has at least $\operatorname{cl}\left(\prod_{2 g} S^{1}\right)=2 g+1$ critical points. This proves assertion (i).

By Theorem 3.4.9 the invariant moduli space $\mathcal{M}_{S U(2)}$ is either empty or a compact closed manifold of the dimension given in Theorem 3.4.9. If this manifold is empty then there is nothing to prove. If it is not empty, we proceed as follows:

In the case $g=0$ the moduli space $\mathcal{M}_{S U(2)}$ of anti-self dual $S U(2)$-invariant connections on the bundle $\Delta^{-}$has dimension 2 . Hence $H^{2}\left(\mathcal{M}_{S U(2)} ; \mathbb{Z}_{2}\right) \cong \mathbb{Z}_{2}$. But $H^{2}\left(\mathcal{B}_{S U(2)} ; \mathbb{Z}_{2}\right)=0$ and hence the inclusion $\mathcal{M}_{S U(2)} \hookrightarrow \mathcal{B}_{S U(2)}$ cannot be a homotopy equivalence. Since the Palais-Smale condition is satisfied, the critical point from (i) cannot lie in the subspace $\mathcal{M}_{S U(2)}$ and hence it cannot be anti-self dual.

The case $g \geq 2$ uses the same argument: Since $\operatorname{dim}\left(\mathcal{M}_{S U(2)}\right)=2(2 g-1)$, we obtain $H^{2(2 g-1)}\left(\mathcal{M}_{S U(2)} ; \mathbb{Z}_{2}\right) \cong \mathbb{Z}_{2}$. Since $\mathcal{B}_{S U(2)} \simeq \prod_{2 g} S^{1}$ and $2(2 g-1)>2 g$, we obtain $H^{2(2 g-1)}\left(\mathcal{B}_{S U(2)} ; \mathbb{Z}_{2}\right)=0$. Hence the inclusion $\mathcal{M}_{S U(2)} \hookrightarrow \mathcal{B}_{S U(2)}$ cannot be a homotopy equivalence and the Palais-Smale condition guarantees the existence of a critical point which does not lie in the subspace $\mathcal{M}_{S U(2)}$. This proves the case $g \geq 2$. The other cases are proved by changing the orientation. This finishes the proof of assertion (ii).

\section{REFERENCES}

[AB] Atiyah, M.F., Bott, R.: The Yang-Mills equation over Riemann surfaces, Phil. Trans. Roy. Soc. London A 308 (1982), 523-615. MR 85k:14006

[AHS] Atiyah, M.F., Hitchin, N.J., Singer, I.M.: Self-duality in four-dimensional Riemannian geometry, Proc. Roy. Soc. London A 362 (1978), 425-461. MR 80d:53023

[AS] Atiyah, M.F., Singer, I.M.: The index of elliptic operators: III, Ann. of Math. 87 (1968), 546-604. MR 38:5245

[BoH] Borel, A., Hirzebruch, F.: Characteristic classes and homogeneous spaces I, Amer. J. Math. 80 (1958), 458-538. MR 21:1586

[Ba] Braam, P.J.: Magnetic monopoles on three-manifolds, J. Differential Geometry 30 (1989), 425-464. MR 90e:53040

[Cho] Cho, Y. S.: Finite group actions on the moduli space of self dual connections I, Trans. Amer. Math. Soc., 323, No. 1 (1991), 233-261. MR 91d:58030

[DK] Donaldson, S.K., Kronheimer, P.B.: "The Geometry of Four-Manifolds", Oxford mathematical monographs, 1990, Oxford University Press. MR 92a:57036

[FU] Freed, D.S., Uhlenbeck, K.K.: "Instantons and Four-Manifolds", MSRI publications, Vol. 1, 1984, New York-Berlin-Heidelberg: Springer Verlag. MR 91i:57019 
[LM] Lawson, H.B. Jr., Michelsohn, M.-L.: "Spin Geometry", Princeton Mathematical Series Vol. 38, 1989, Princeton University Press. MR 91g:53001

[MS] Milnor, J.W., Stasheff, J.D.: "Characteristic Classes", Annals of Mathematical Studies No. 76, 1974, Princeton University Press. MR 55:13428

[Pal1] Palais, R.S.: Lusternik-Schnirelman Theory on Banach Manifolds, Topology Vol. 5 (1966), 115-132. MR 41:4584

[Pal2] Palais, R.S.: The Principle of Symmetric Criticality, Comm. Math. Phys. 69 (1979), 19-30. MR 81c:58026

[Pa1] Parker, T.H.: A Morse theory for equivariant Yang-Mills, Duke Mathematical Journal, Vol. 66, No. 2 (1992), 337-356. MR 93e:58031

[Pa2] Parker, T.H.: Non-minimal Yang-Mills fields and dynamics, Invent. Math. 107 (1992), 397-420. MR 93b:58037

[Rå] Råde, J.: Compactness Theorems for Invariant Connections, submitted for publication.

[Se] Segal, G.B.: Equivariant K-theory, Publ. Math. Inst. Hautes Études Sci. 34 (1968), 129-151. MR 38:2769

[SS] Sadun, L., Segert, J.: Non-self-dual Yang-Mills connections with quadrupole symmetry, Comm. Math. Phys. 145 (1992), 363-391. MR 94b:53056

[SSU] Sibner, L.M., Sibner, R.J., Uhlenbeck, K.K. : Solutions to Yang-Mills equations that are not self-dual, Proc. Natl. Acad. Sci. USA Vol. 86 (1989), 8610-8613. MR 90j:58032

[Wa] Wasserman, A.G.: Equivariant differential topology, Topology Vol. 8 (1969), 127-150. MR 40:3563

[Wan] Wang, H.-Y.: The existence of nonminimal solutions to the Yang-Mills equation with group $S U(2)$ on $S^{2} \times S^{2}$ and $S^{1} \times S^{3}$, J. Differential Geometry Vol. 34 (1991), 701-767. MR 93b:58036

Department of Pure Mathematics and Mathematical Statistics, University of CamBridge, 16 Mill Lane, Cambridge, CB2 1SB, U.K.

Current address: Department of Mathematics, University of California, Berkeley, Evans Hall, Berkeley, California 94720-3840

E-mail address: ursula@math.berkeley.edu 